\title{
Off-axis beaming from subwavelength apertures
}

Humeyra Caglayan, Irfan Bulu, and Ekmel Ozbay

Citation: Journal of Applied Physics 104, 073108 (2008);

View online: https://doi.org/10.1063/1.2990063

View Table of Contents: http://aip.scitation.org/toc/jap/104/7

Published by the American Institute of Physics

\section{Articles you may be interested in}

Off-axis directional beaming via photonic crystal surface modes Applied Physics Letters 92, 092114 (2008); 10.1063/1.2842424

Off-axis directional beaming of optical field diffracted by a single subwavelength metal slit with asymmetric dielectric surface gratings

Applied Physics Letters 90, 051113 (2007); 10.1063/1.2437730

Frequency scanning non-diffraction beam by metasurface

Applied Physics Letters 110, 031108 (2017); 10.1063/1.4974210

Reconfigurable conversions of reflection, transmission, and polarization states using active metasurface Applied Physics Letters 110, 121901 (2017); 10.1063/1.4979033

Optical beam focusing by a single subwavelength metal slit surrounded by chirped dielectric surface gratings Applied Physics Letters 92, 013103 (2008); 10.1063/1.2828716

Cascaded metasurfaces for complete phase and polarization control

Applied Physics Letters 102, 231116 (2013); 10.1063/1.4810873

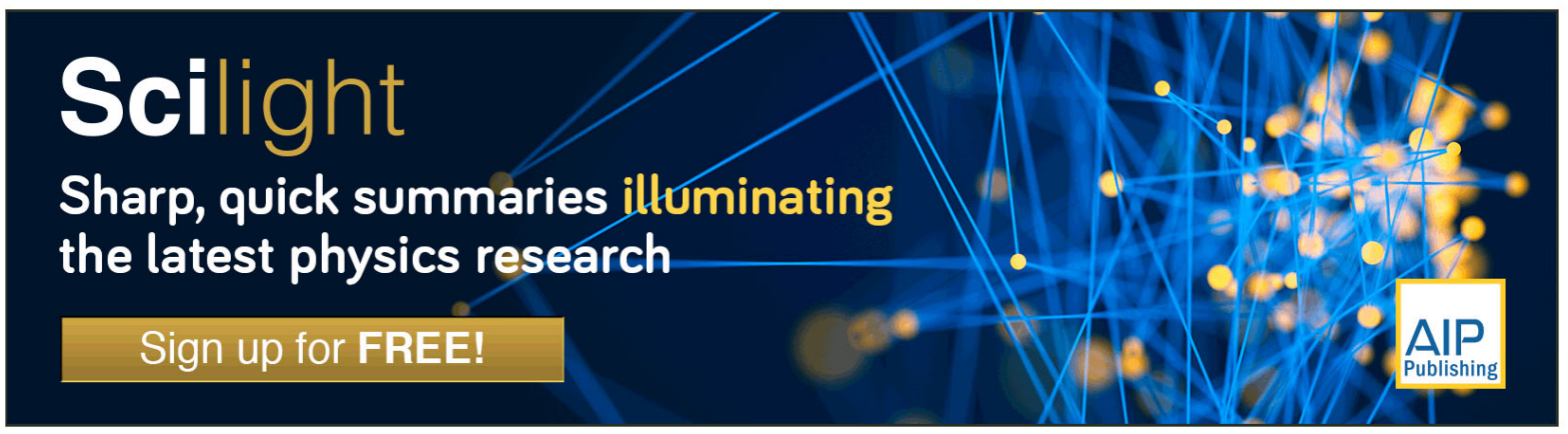




\title{
Off-axis beaming from subwavelength apertures
}

\author{
Humeyra Caglayan, ${ }^{1, a)}$ Irfan Bulu, ${ }^{1,2}$ and Ekmel Ozbay ${ }^{1}$ \\ ${ }^{1}$ Nanotechnology Research Center-NANOTAM, Department of Physics, Department of Electrical and \\ Electronics Engineering, Bilkent University, Bilkent, 06800 Ankara, Turkey \\ ${ }^{2}$ School of Engineering and Applied Sciences, Harvard University, 33 Oxford Street, Cambridge, \\ Massachusetts 02138, USA
}

(Received 20 May 2008; accepted 13 August 2008; published online 3 October 2008)

\begin{abstract}
Photonic crystal waveguides and metallic subwavelength apertures are promising tools for light manipulation. It is possible to obtain enhanced directional beams by using these structures via coupling to surface waves. In addition, these apertures can be designed to steer such directional beams by introducing asymmetrical gratings on the output surface. In the present paper, we report directional yet off-axis beaming from subwavelength apertures at microwave frequencies. The full width at half maximum of the beam is $10^{\circ}$ while the beaming angle is $15^{\circ}$. Our results show that it is possible to steer the beam by the appropriate modification of the output surface. (C) 2008 American Institute of Physics. [DOI: 10.1063/1.2990063]
\end{abstract}

Manipulation of light within very small volumes is very important for technological applications. ${ }^{1}$ Photonic crystal (PC) waveguides and metallic subwavelength apertures are promising tools for this purpose. However, there are two main constraints for subwavelength apertures: poor transmission and diffraction. It is possible to overcome these limitations by changing the surface of the apertures. Although electromagnetic (EM) waves transmitted from a subwavelength aperture diffract in all directions, a metallic aperture surrounded with periodic grooves can enhance transmission and channel the beam into a narrow spatial region. ${ }^{2-8}$ Similarly, one would be able to obtain an enhanced as well as a directional beam from a subwavelength PC waveguide with an interface layer. ${ }^{9-11}$ The enhanced beam from these apertures is directed in the propagation direction. ${ }^{12}$ However, it is also important to change the beam angle. Recently, it has been shown that steering a beam is possible by designing the periods asymmetrically on different sides of the aperture. $^{13-15}$ In the present paper, we investigated off-axis beaming from both a metallic subwavelength aperture and $\mathrm{PC}$ waveguide in the microwave regime. The output surfaces are designed asymmetrically to steer the beaming angle.

Surface waves or surface plasmons (SPs) are the collective longitudinal excitation of electrons. Since SPs have a longer wavelength than light, one way to excite SPs is to have periodic structures on the surface to satisfy the energy and momentum conservation. ${ }^{16}$ At microwave frequencies metals have skin depth approaching to zero and thus behave as perfect electric conductor. Perfect electric conductors do not support SPs anyhow. The existence of SPs at corrugated perfect metal surfaces is due to the corrugation, and therefore, they are frequently referred to as "spoof" $\mathrm{SPs}^{17}$ or "designer" SPs. ${ }^{18}$ Therefore, we dressed the input surface of our metallic $(\mathrm{Al})$ subwavelength $(\lambda / 10)$ structure with periodic grooves (Fig. 1). We have chosen $p$-polarization because spoof SPs, just like natural SPs, do not exist for $s$-polarized

\footnotetext{
a) Author to whom correspondence should be addressed. Electronic addresses: caglay@bilkent.edu.tr and caglayan@fen.bilkent.edu.tr.
}

incident waves. ${ }^{19}$ The period of the input grooves is $16 \mathrm{~mm}$, which possesses SP resonance at $14.5 \mathrm{GHz} .{ }^{20}$ While the input surface gratings allow for coupling to SP, the output surface affects the beam shape. The beam diffracts if no periodic structures are on the output surface. On the other hand, beaming occurs when the output side of the subwavelength aperture has periodic grooves. ${ }^{3}$ In order to change the beaming angle or, in other words, to steer the beam, we have to redesign the output surface grooves. We changed the periods of the grooves on different sides of the aperture. One of the sides has a smaller period $(14 \mathrm{~mm})$ while the other side has a longer period $(22 \mathrm{~mm})$ than the input side.

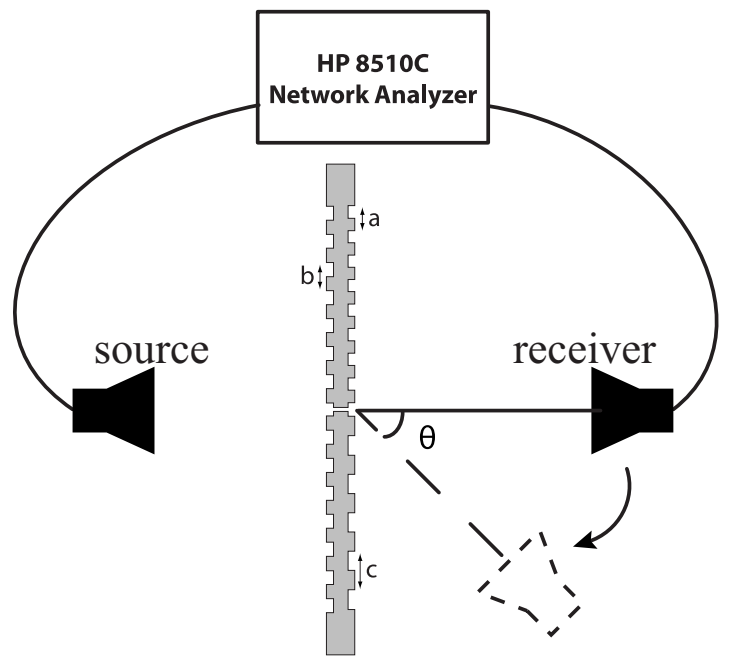

FIG. 1. The metallic (Al) grating structure has a subwavelength $(2 \mathrm{~mm}$ $=\lambda / 10)$ slit at the center and the height of the grating indentations is $4 \mathrm{~mm}$. The input surface grating period is $b=16 \mathrm{~mm}$ in order to couple the SPs. The outsurface has different grating periods $(a=14 \mathrm{~mm}$ and $c=22 \mathrm{~mm})$. The height of the structure is $30 \mathrm{~cm}(\approx 15 \lambda)$, which is enough for practical purposes; the system behaves as having infinite height in the normal to the plane of the experimental setup. The experimental setup consists of a Hewlett Packard 8510C network analyzer and two standard-gain horn antennae in order to measure the transmission amplitude. The radiation is normally incident upon the sample from $15 \mathrm{~cm}$ by the source antenna. The receiver antenna was placed $100 \mathrm{~cm}(\approx 50 \lambda)$ away from the sample's back face and was connected to a rotating arm in order to measure the angular dependence of the radiation. 


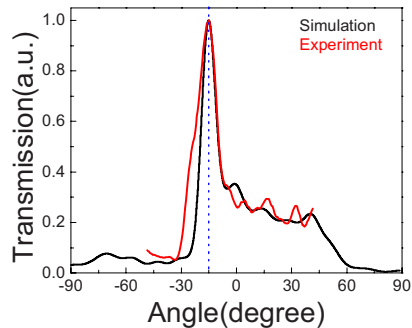

(a)

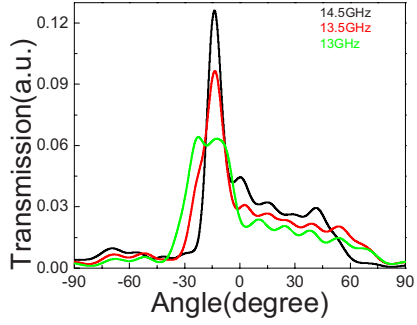

(b)
FIG. 2. (Color online) (a) The angular distribution of the transmission from the asymmetric subwavelength grating aperture possesses off-axis directional beaming with a FWHM of $10^{\circ}$ and a beaming angle of $15^{\circ}$ at 14.5 $\mathrm{GHz}(20.7 \mathrm{~mm})$. The FDTD calculations are in agreement with the experiment. (b) The angular distribution transmission from the asymmetric subwavelength grating aperture for different frequencies is presented. The beaming could not be observed for the frequencies away from the existence of SP.

The experimental setup consisted of an HP 8510C network analyzer and two standard-gain horn antennae in order to measure the transmission amplitude. Radiation was normally incident upon the sample from $15 \mathrm{~cm}$ by a source antenna. The receiver antenna was $100 \mathrm{~cm}$ away from the sample (Fig. 1). Measurements were performed in the microwave spectrum of $10-18 \mathrm{GHz}$, corresponding to a wavelength region of $16.7-30 \mathrm{~mm}$. The measured angular distribution of the transmission from the subwavelength aperture at $14.5 \mathrm{GHz}(20.7 \mathrm{~mm})$ is shown in Fig. 2(a). We observed off-axis directional beaming with a full width at half maximum (FWHM) of $10^{\circ}$ and a beaming angle of $15^{\circ}$. Beaming angle is the angle between the exit beam and the waveguide channel. The finite difference time domain (FDTD) (Ref. 21) calculations are in agreement with the experiment. Figure 2(b) shows the angular distribution for SP resonance frequency and frequencies away from the resonance. The beaming could not be observed for the frequencies away from the existence of SP.

When a $p$-polarized EM wave, which is incident to the metallic surface induced surface waves, flows through the aperture via grating, diffraction beaming phenomena occur. The SP dispersion relation and grating equation state that

$$
k_{\mathrm{sp}} \pm N k_{g}=k_{0} \sin \theta=k_{0 \|},
$$

where $k_{\mathrm{sp}}$ is the wave vector of SP, $k_{0 \|}$ is the portion of the incident wave vector that is in the plane of the metal, $N$ is an integer, and $k_{g}=2 \pi / \lambda_{g}$ is the grating wave vector where $\lambda_{g}$ is the grating period.

The possibility to have steered beaming is a combinatory result of the generalized form for the conservation of the parallel component of the wave vector at the interface of a periodic medium and the finite angle span of the source. Finite width sources are never perfectly collimated. Thus, the input beam has a small angular span that gives $k_{\text {in } \|}= \pm k_{0 \|}$, which is small yet nonzero. The existence of this small $k_{0 \|}$ is necessary to obtain the steered beaming phenomenon. This is because if $k_{\text {inl }}=0$, it would be impossible to satisfy $k_{\| \text {out }}($ $\neq 0)=k_{\mathrm{sp}}+k_{g}=k_{\mathrm{in} \|}=0$. At the interface of a homogeneous medium the parallel component of the wave vector is conserved $\left(k_{\text {in } \|}=k_{\text {out } \|}\right)$. At the interface of a periodic medium the parallel
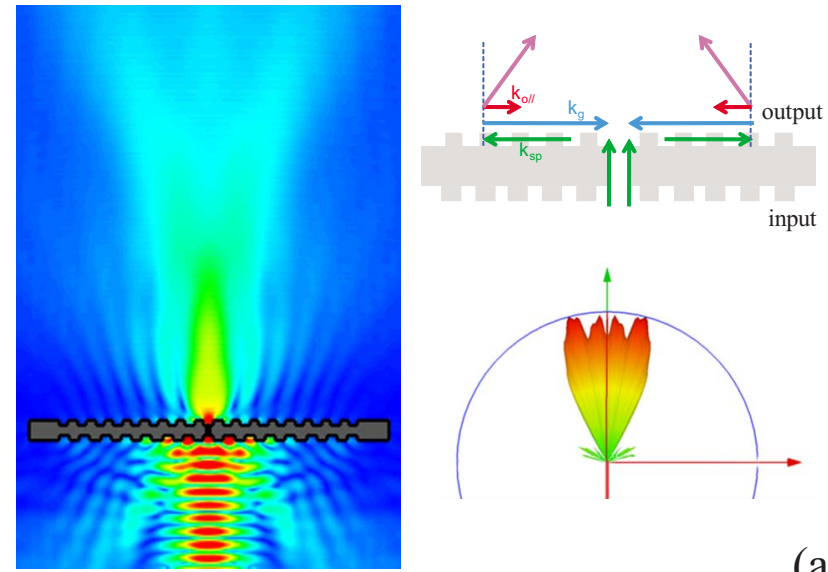

(a)
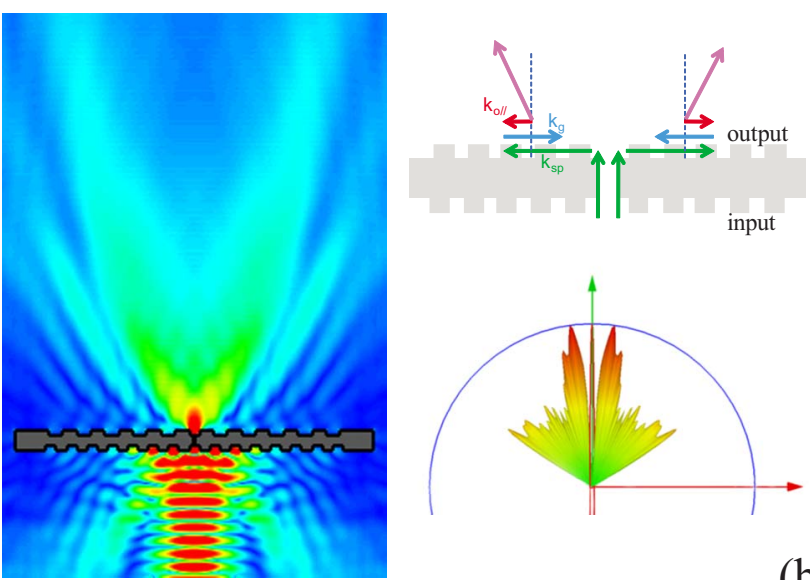

(b)
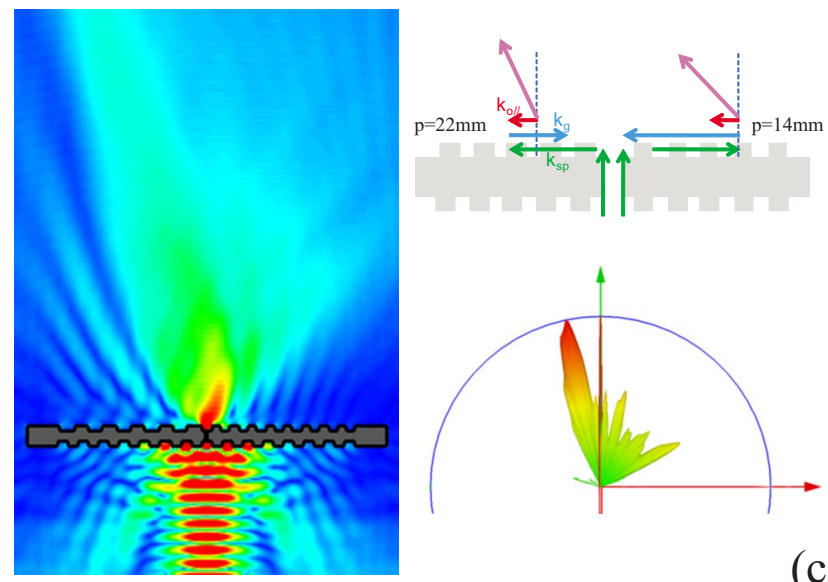

(c)

FIG. 3. (Color online) Calculated $E$-field and far field for subwavelength apertures with an input side grating period of $16 \mathrm{~mm}$. (a) The projected direction of the diffracted beam is toward the waveguide channel for a structure with an output surface grating period of $14 \mathrm{~mm}$. (b) The projected direction of the diffracted beam is away from the waveguide channel for a structure with an output surface grating period of $22 \mathrm{~mm}$. (c) The off-axis beam was achieved with the combination of these structures (output surface gratings are 14 and $22 \mathrm{~mm}$ on the different sides of the aperture). The sign convention of the diffracted angles is also shown for every structure.

component of the wave vector is conserved within a reciprocal lattice vector corresponding to the periodicity of the surface $\left(k_{\text {in } \|}=k_{\text {out } \|}+N k_{g}\right)$. The demonstrated steered beaming (beaming angle different than zero) is always an umklapp 


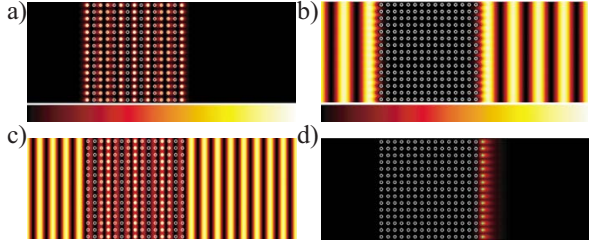

FIG. 4. (Color online) Electric field intensity profiles of the modes supported by the finite size PC: (a) mode decays in the air but extends in the PC, (b) mode extends in the air but decays in the PC, and (c) mode extends in the air and in the PC. (d) Surface mode: decays both in the air and in the PC; it is localized at the modified interface layer. These field profiles are calculated by using the plane wave expansion method.

process, i.e., a process with, and in particular, an umklapp process with $N= \pm 1$. The beaming angle would be restricted by the angle span of the source.

When the grating vector $k_{g}$ is larger than $k_{\mathrm{sp}}$, the in-plane component $k_{0||}$ is negative. Therefore, the projected direction of the diffracted beam is toward the waveguide channel since the grating vector $k_{g}$ is smaller than $k_{\mathrm{sp}}$ [Fig. 3(a)]. On the other hand, when the grating vector $k_{g}$ is larger than $k_{\mathrm{sp}}$, the beaming angle is away from the waveguide channel [Fig. $3(b)]$. The combinations of these grating vectors on the different sides of the subwavelength aperture will possess offaxis beaming [Fig. 3(c)].

PCs possess a rather different structure and properties than subwavelength metallic apertures. However, a metallic surface and the surface of a corrugated PC have in common the fact that both surfaces can support surface propagating EM waves. ${ }^{22}$ Therefore, it is possible to use a PC waveguide for similar applications via exciting the surface modes. The finite size PC can support three types of modes: ${ }^{23}$ (1) mode decays in the air but extends in the PC, (2) mode extends in the air but decays in the PC, and (3) mode extends in the air and in the PC. The intensity profiles of these modes, which are calculated using plane wave expansion method, ${ }^{24}$ are shown in Figs. 4(a)-4(c). In addition to these modes, it is also possible to obtain one more mode by the modification of the interface layer. This mode decays both in air and in the PC; it is localized at the modified interface layer [Fig. 4(d)]. However, these modes have real wave vectors parallel to the PC surface. As a result, these modes are surface propagating waves.

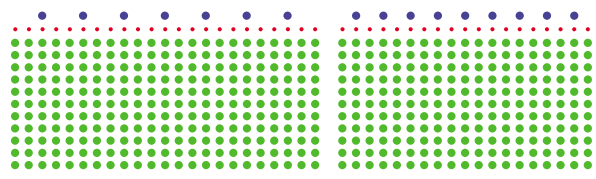

FIG. 5. (Color online) The $2 \mathrm{D}$ PC is constructed from a $43 \times 11$ square array of circular alumina rods (indicated as green dots). The crystal is 11 layers along the propagation direction. The radius of the rods is $1.55 \mathrm{~mm}$ and the dielectric constant of the alumina is 9.61. The separation between the center of the rods along the lattice of the vectors is $a=11 \mathrm{~mm}$. This PC has a band gap of between 8.7 and $13.2 \mathrm{GHz}$. The radius of the rods in the modified layer (indicated as red dots) is half of the regular rods $(0.76 \mathrm{~mm})$. This layer creates surface propagating modes in the band gap. The rods in the gratinglike layer have equal radii of the bulk PC rods. The asymmetric gratinglike layer has a double period $(22 \mathrm{~mm})$ on one side and a triple period $(33 \mathrm{~mm})$ on the other side of the PC waveguide.

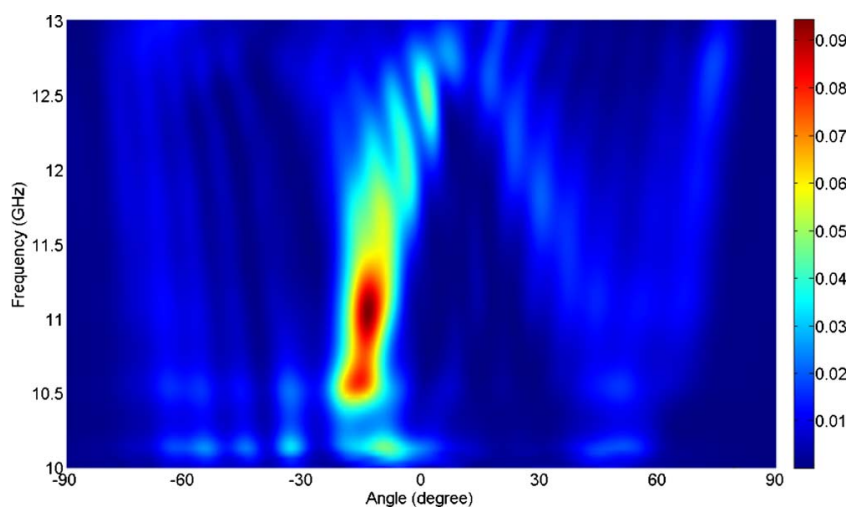

FIG. 6. (Color online) FDTD calculations of the transmission from the PC waveguide exhibit off-axis beaming around $11 \mathrm{GHz}$. The periods of the gratinglike layer on the different sides of the waveguide were designed as 22 and $33 \mathrm{~mm}$ in order to steer the beaming angle.

The PC that was used in the present paper was a twodimensional (2D) PC constructed from an 11 layer square array of circular dielectric rods along the propagation direction. The separation between the center of the rods along the lattice of the vectors was $a=11 \mathrm{~mm}$. The radius of the alumina rods was $1.55 \mathrm{~mm}$ and the dielectric constant of the alumina was 9.61. This PC has a band gap between 8.7 and 13.2 GHz. In order to obtain a line defect that is similar to a subwavelength aperture, one line of the $\mathrm{PC}$ is removed. One layer of rods with a radius of $0.76 \mathrm{~mm}$ is added to this $\mathrm{PC}$ waveguide to take advantage of the surface propagating modes (Fig. 5). Since the excited surface modes are evanescent and cannot couple to the radiating modes of the free space, an extra layer (gratinglike layer) is needed in front of the modified layer of the PC.

The gratinglike layer is identical to the gratings on the output surface of the metallic structures. The EM waves throughout the waveguide diffract from this layer. The momentum of the periodic corrugation surface waves and the angle span of the finite source define the beaming angle. Therefore, to steer the beaming angle period of the gratinglike layer, the different sides of the waveguide have to be different. In the present paper, we designed the grating periods as 22 and $33 \mathrm{~mm}$. FDTD calculations of the transmission

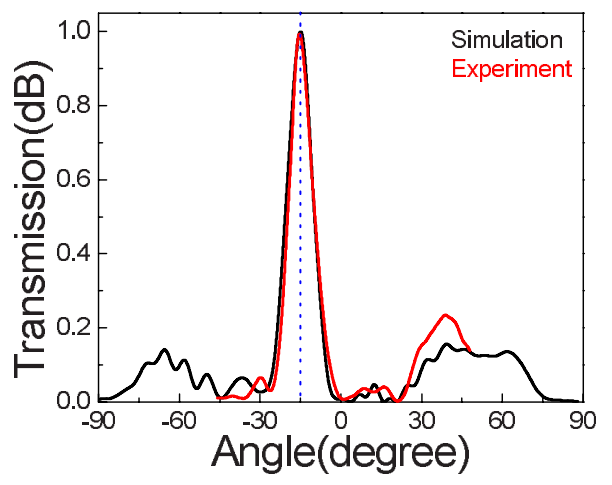

FIG. 7. (Color online) The angular distribution of the transmission from the PC waveguide with different gratinglike layer periods on different sides of the waveguide possesses off-axis directional beaming with a FWHM of $10^{\circ}$ and a beaming angle of $15^{\circ}$ at $11 \mathrm{GHz}(27 \mathrm{~mm})$. The FDTD calculations are in agreement with the experiment. 
from this PC waveguide exhibit off-axis beaming around 11 $\mathrm{GHz}$ (Fig. 6). We used the same simulation tools and experimental setup for this part as in the metallic structures, but we used $s$-polarization ( $E$-field is parallel to the rods). The FWHM of the beam is $10^{\circ}$ with a beaming angle of $15^{\circ}$ at 11 $\mathrm{GHz}(27 \mathrm{~mm})$. This is in good agreement with the calculations (Fig. 7). The beam is steered by arranging grating periods asymmetrically.

In conclusion, it is possible to change the beam by using subwavelength metallic apertures and a PC waveguide via coupling to the surface waves. The gratings have to be arranged asymmetrically in order to steer the beam. We observed a beaming angle of $15^{\circ}$ with a FWHM of $10^{\circ}$. The momentum of the periodic corrugation surface waves and the angle span of the finite source define the beaming angle. Our results show that it is possible to steer the beam by the appropriate modification of the output surface.

This work is supported by the European Union under the projects EU-METAMORPHOSE, EU-PHOREMOST, EUPHOME, EU-ECONAM, and TUBITAK under Project Nos. 105E066, 105A005, 106E198, 106A017, and 107A012. One of the authors (E.O.) also acknowledges partial support from the Turkish Academy of Sciences.

${ }^{1}$ E. Ozbay, Science 311, 189 (2006).

${ }^{2}$ H. J. Lezec, A. Degiron, E. Devaux, R. A. Linke, L. Martin-Moreno, F. J. Garcia-Vidal, and T. W. Ebbesen, Science 297, 820 (2002).

${ }^{3}$ H. Caglayan, I. Bulu, and E. Ozbay, J. Opt. Soc. Am. B 23, 419 (2006).

${ }^{4}$ F. J. Garcia-Vidal, L. Martin-Moreno, H. J. Lezec, and T. W. Ebbesen, Appl. Phys. Lett. 83, 4500 (2003).
${ }^{5}$ S. S. Akarca-Biyikli, I. Bulu, and E. Ozbay, J. Opt. A, Pure Appl. Opt. 7, S159 (2005).

${ }^{6}$ M. J. Lockyear, A. P. Hibbins, J. R. Sambles, and C. R. Lawrence, Appl. Phys. Lett. 84, 2040 (2004)

${ }^{7}$ L. Martin-Moreno, F. J. Garcia-Vidal, H. J. Lezec, A. Degiron, and T. W. Ebbesen, Phys. Rev. Lett. 90, 167401 (2003).

${ }^{8}$ Y. Poujet, M. Roussey, J. Salvi, F. I. Baida, D. Van Labeke, A. Perentes, C. Santschi, and P. Hoffmann, Photonics Nanostruct. Fundam. Appl. 4, 47 (2006).

${ }^{9}$ E. Moreno, F. J. Garcia-Vidal, and L. Martin-Moreno, Phys. Rev. B 69, 121402 (2004).

${ }^{10}$ I. Bulu, H. Caglayan, and E. Ozbay, Opt. Lett. 30, 3078 (2005).

${ }^{11}$ P. Kramper, M. Agio, C. M. Soukoulis, A. Birner, F. Muller, R. B. Wehrspohn, U. Gosele, and V. Sandoghdar, Phys. Rev. Lett. 92, 113903 (2004).

${ }^{12}$ E. Moreno, L. Martín-Moreno, and F. J. García-Vidal, Photonics Nanostruct. Fundam. Appl. 2, 97 (2004).

${ }^{13}$ S. Kim, H. Kim, Y. Lim, and B. Lee, Appl. Phys. Lett. 90, 051113 (2007).

${ }^{14}$ D. Z. Lin, T.-D. Cheng, C.-K. Chang, J.-T. Yeh, J.-M. Liu, C.-S. Yeh, and C.-K. Lee, Opt. Express 15, 2585 (2007).

${ }^{15}$ H. Caglayan, I. Bulu, and E. Ozbay, Appl. Phys. Lett. 92, 092114 (2008).

${ }^{16}$ H. Raether, Surface Plasmons on Smooth and Rough Surfaces and on Gratings (Springer-Verlag, Berlin, 1988).

${ }^{17}$ J. B. Pendry, L. Martín-Moreno, and F. J. Garcia-Vidal, Science 305, 847 (2004).

${ }^{18}$ A. P. Hibbins, B. R. Evans, and J. R. Sambles, Science 308, 670 (2005).

${ }^{19}$ E. Moreno, L. Martín-Moreno, and F. J. García-Vidal, J. Opt. A, Pure Appl. Opt. 8, S94 (2006).

${ }^{20}$ H. Caglayan, I. Bulu, and E. Ozbay, Opt. Express 13, 1666 (2005).

${ }^{21}$ A. Taflove and S. Hagness, Computational Electrodynamics: The FiniteDifference Time-Domain Method (Artech House, Boston, 2000).

${ }^{22}$ B. Wang, W. Dai, A. Fang, L. Zhang, G. Tuttle, Th. Koschny, and C. M. Soukoulis, Phys. Rev. B 74, 195104 (2006).

${ }^{23}$ J. D. Joannopoulos, R. D. Meade, and J. N. Winn, Photonic Crystal: Molding the Flow of Light (Princeton University Press, Princeton, NJ, 1995).

${ }^{24}$ K. M. Ho, C. T. Chan, and C. M. Soukoulis, Phys. Rev. Lett. 65, 3152 (1990). 